\title{
Effects of the arrival of fresh organic matter on eroded and nutrient- depleted trawling grounds (Gulf of Castellammare, SW Mediterranean)
}

Sarah Paradis ${ }^{1}$, Antonio Pusceddu ${ }^{2}$, Pere Masqué ${ }^{1,3,4,5}$, Pere Puig ${ }^{6}$, Davide Moccia ${ }^{2}$, Tommaso Russo ${ }^{7}$, 5 Claudio Lo Iacono 6,8

${ }^{1}$ Institut de Ciència i Tecnologia Ambientals, Universitat Autònoma de Barcelona, Bellaterra, 08193, Spain

${ }^{2}$ Dipartimento di Scienze della Vita e dell'Ambiente, Università degli Studi di Cagliari, Cagliari, 09126, Italy

${ }^{3}$ Departament de Física, Universitat Autònoma de Barcelona, Bellaterra, 08193, Spain

${ }^{4}$ School of Natural Sciences, Centre for Marine Ecosystems Research, Edith Cowan University, Joondalup, WA 6027,

10 Australia

${ }^{5}$ School of Physics and Oceans Institute, University of Western Australia, Crawley, WA 6009, Australia

${ }^{6}$ Marine Sciences Institute, Consejo Superior de Investigaciones Científicas, Barcelona, 08003, Spain

${ }^{7}$ Laboratory of Experimental Ecology and Aquaculture, Department of Biology, University of Rome Tor Vergata, Rome, 00133, Italy

$15{ }^{8}$ National Oceanography Centre, University of Southampton Waterfront Campus, Southampton, SO14 3ZH, United Kingdom

Correspondence to: Sarah Paradis (sarah.paradis@uab.cat)

Supplementary Information

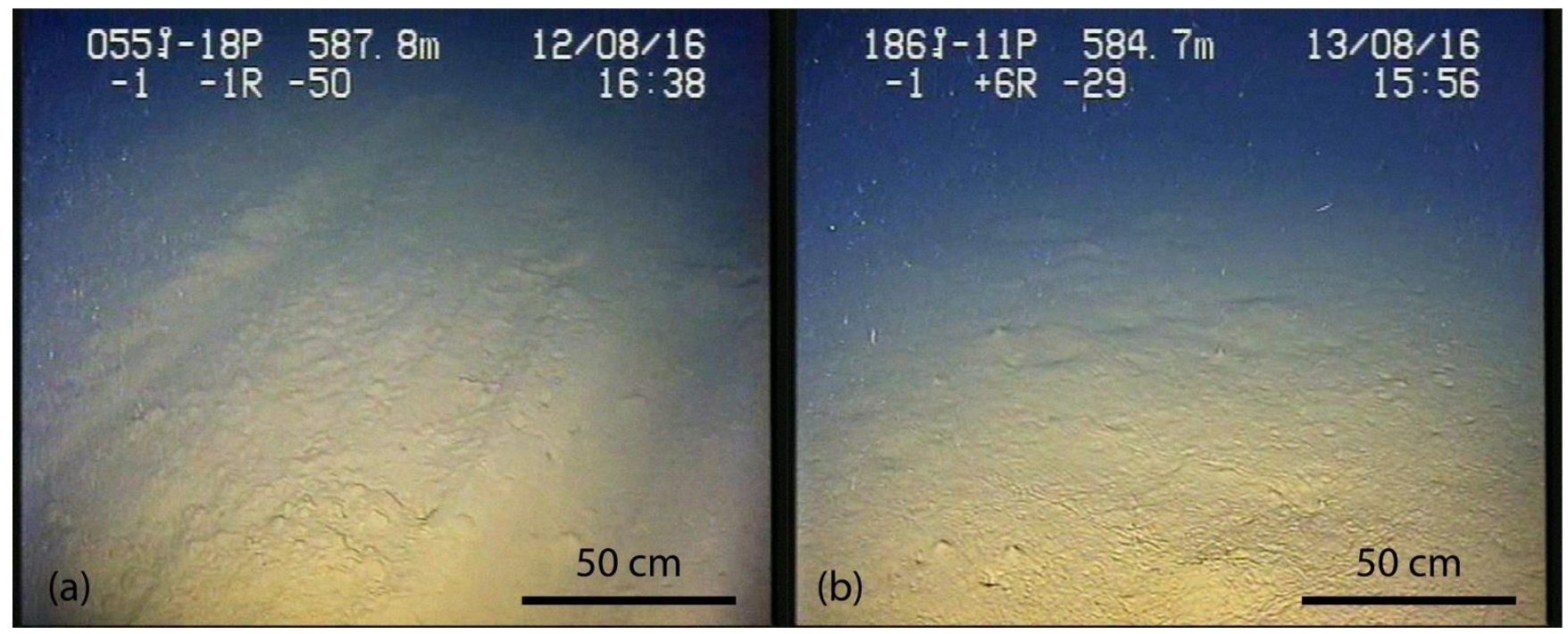

Figure S1. ROV-seafloor pictures from the trawled (a) and untrawled (b) sites in the Gulf of Castellammare. The trawled site presents

20 linear marks and furrows on eroded sediments produced by the intense trawling activity in the area, whereas the untrawled site shows mounds and tracks characteristic of bioturbation activity. 


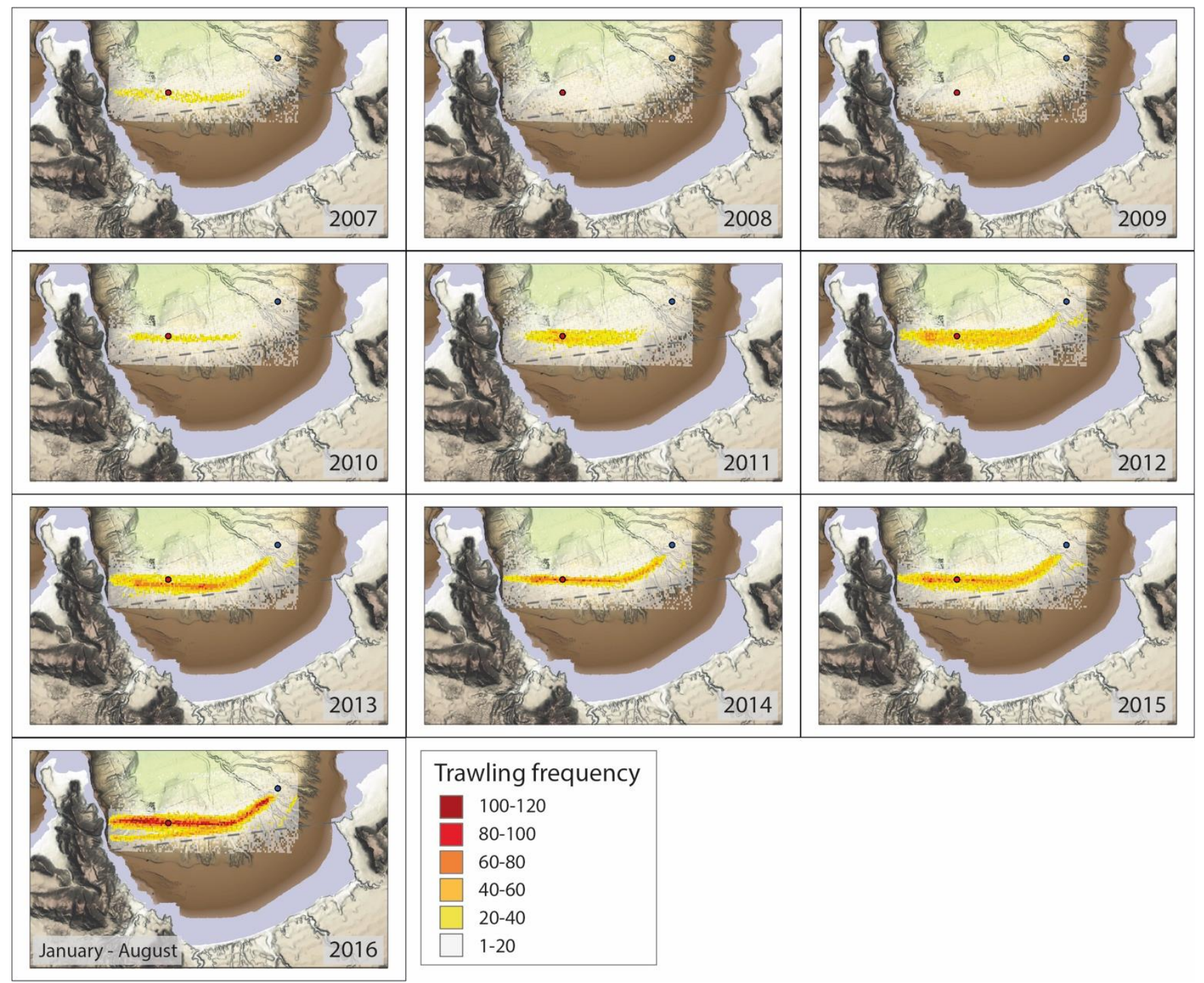

Figure S2. Evolution of trawling intensity. Number of times trawled per year and per grid cell (200 x $200 \mathrm{~m})$ in the Gulf of Castellammare. Note the increasing trawling frequency since 2007, with the exception of 2008 and 2009, and that computation of trawling frequency in 2016 is limited to VMS data prior to the sampling period, August $10^{\text {th }}$. Blue and red circles indicate the sampling sites of the untrawled, and 5 trawled site, respectively. 
Table S1. Results of the PERMANOVA test for differences in organic matter quantity (protein, carbohydrate, lipid, biopolymeric C, and phytopigment) between trawled and untrawled sites, depth layers, and their interaction. The percentage variance explained by each source of variation is also reported. $\mathrm{df}=$ degrees of freedom; $\mathrm{MS}=$ mean square; Pseudo-F = permutational $\mathrm{F}$; significance of test obtained from Monte Carlo simulations: $*=p(\mathrm{MC})<0.05 ; * *=p(\mathrm{MC})<0.01$; *** $=p(\mathrm{MC})<0.001$; n.s. $=$ not significant.

\begin{tabular}{|c|c|c|c|c|c|c|}
\hline Variable & Source & df & MS & Pseudo-F & $p(\mathrm{MC})$ & Explained variance $(\%)$ \\
\hline \multirow[t]{5}{*}{ Protein $\left(\mathrm{mgC} \cdot \mathrm{g}^{-1}\right)$} & Site & 1 & 4.62 & 13.2 & $* *$ & 22 \\
\hline & Depth & 4 & 3.51 & 10.1 & $* * *$ & 40 \\
\hline & Site x Depth & 4 & 0.82 & 2.4 & n.s. & 12 \\
\hline & Residual & 20 & 0.35 & & & 27 \\
\hline & Total & 29 & & & & \\
\hline \multirow[t]{5}{*}{ Carbohydrate $\left(\mathrm{mgC} \cdot \mathrm{g}^{-1}\right)$} & Site & 1 & 11.29 & 47.9 & $* * *$ & 44 \\
\hline & Depth & 4 & 1.68 & 7.1 & $* *$ & 15 \\
\hline & Site x Depth & 4 & 1.57 & 6.6 & $* *$ & 27 \\
\hline & Residual & 20 & 0.23 & & & 14 \\
\hline & Total & 29 & & & & \\
\hline \multirow[t]{5}{*}{ Lipid $\left(\mathrm{mgC} \cdot \mathrm{g}^{-1}\right)$} & Site & 1 & 4.68 & 20.4 & $* * *$ & 23 \\
\hline & Depth & 4 & 4.73 & 20.6 & $* * *$ & 59 \\
\hline & Site x Depth & 4 & 0.20 & 0.9 & n.s. & 0 \\
\hline & Residual & 20 & 0.23 & & & 18 \\
\hline & Total & 29 & & & & \\
\hline \multirow[t]{5}{*}{ Biopolymeric $\mathrm{C}\left(\mathrm{mgC} \cdot \mathrm{g}^{-1}\right)$} & Site & 1 & 13.02 & 36.9 & $* * *$ & 55 \\
\hline & Depth & 4 & 1.36 & 3.9 & $*$ & 11 \\
\hline & Site x Depth & 4 & 0.87 & 2.5 & n.s. & 11 \\
\hline & Residual & 20 & 0.35 & & & 23 \\
\hline & Total & 29 & & & & \\
\hline \multirow[t]{5}{*}{ Phytopigment $\left(\mu \mathrm{gC} \cdot \mathrm{g}^{-1}\right)$} & Site & 1 & 11.25 & 93.0 & $* * *$ & 50 \\
\hline & Depth & 4 & 3.53 & 29.1 & $* * *$ & 38 \\
\hline & Site x Depth & 4 & 0.29 & 2.4 & n.s. & 4 \\
\hline & Residual & 20 & 0.12 & & & 8 \\
\hline & Total & 29 & & & & \\
\hline
\end{tabular}


Table S2. Results of the pairwise comparison testing for differences in the quantity of each investigated variable between trawled and untrawled sites at each depth. $\mathrm{df}=$ degrees of freedom; MS = mean square; Pseudo-F = permutational F; significance of test obtained from Monte Carlo simulations: $*=p(\mathrm{MC})<0.05 ; * *=p(\mathrm{MC})<0.01 ; * * *=p(\mathrm{MC})<0.001$; n.s. $=$ not significant.

Pair-wise comparisons

Factor: Trawled vs. Untrawled

\begin{tabular}{|c|c|c|c|}
\hline Variable & Depth $(\mathrm{cm})$ & $\mathrm{t}$ & $p(\mathrm{MC})$ \\
\hline \multirow[t]{5}{*}{ Protein $\left(\mathrm{mgC} \cdot \mathrm{g}^{-1}\right)$} & $0-1$ & 0.502 & n.s. \\
\hline & $1-3$ & 1.236 & n.s. \\
\hline & $3-5$ & 1.871 & n.s. \\
\hline & $5-7$ & 2.94 & $*$ \\
\hline & $7-9$ & 1.799 & n.s. \\
\hline \multirow[t]{5}{*}{ Carbohydrate $\left(\mathrm{mgC} \cdot \mathrm{g}^{-1}\right)$} & $0-1$ & 3.648 & $*$ \\
\hline & $1-3$ & 9.139 & $* *$ \\
\hline & $3-5$ & 2.997 & $*$ \\
\hline & $5-7$ & 0.643 & n.s. \\
\hline & $7-9$ & 1.06 & n.s. \\
\hline \multirow[t]{5}{*}{ Lipid $\left(\mathrm{mgC} \cdot \mathrm{g}^{-1}\right)$} & $0-1$ & 0.666 & n.s. \\
\hline & $1-3$ & 3.753 & $*$ \\
\hline & $3-5$ & 1.044 & n.s. \\
\hline & $5-7$ & 2.921 & $*$ \\
\hline & $7-9$ & 5.63 & $* *$ \\
\hline \multirow[t]{5}{*}{ Biopolymeric $\mathrm{C}\left(\mathrm{mgC} \cdot \mathrm{g}^{-1}\right)$} & $0-1$ & 2.058 & n.s. \\
\hline & $1-3$ & 4.197 & $*$ \\
\hline & $3-5$ & 2.737 & n.s. \\
\hline & $5-7$ & 3.105 & $*$ \\
\hline & $7-9$ & 3.783 & $*$ \\
\hline \multirow[t]{5}{*}{ Phytopigment $\left(\mu \mathrm{gC} \cdot \mathrm{g}^{-1}\right)$} & $0-1$ & 1.846 & n.s. \\
\hline & $1-3$ & 6.128 & $* *$ \\
\hline & $3-5$ & 11.417 & $* * *$ \\
\hline & $5-7$ & 4.572 & $*$ \\
\hline & $7-9$ & 3.251 & $*$ \\
\hline
\end{tabular}

Table S3. Results of the PERMANOVA testing for differences in biochemical composition (protein, carbohydrate, lipid and phytopigment 5 contents) between trawled and untrawled sites, depth layers, and their interaction. The percentage variance explained by each source of variation is also reported. $\mathrm{df}=$ degrees of freedom; $\mathrm{MS}=$ mean square; Pseudo-F = permutational F; significance of test obtained from Monte Carlo simulations: $*=p(\mathrm{MC})<0.05 ; * *=p(\mathrm{MC})<0.01$; $* * *=p(\mathrm{MC})<0.001$; n.s. $=$ not significant.

\begin{tabular}{lllllll} 
& Source & df & MS & Pseudo-F & $p(\mathrm{MC})$ & Explained variance $(\%)$ \\
\hline Biochemical composition & Site & 1 & 31.9 & 34.0 & $* * *$ & 36 \\
& Depth & 1 & 13.5 & 14.4 & $* * *$ & 36 \\
& Site x Depth & 4 & 2.9 & 3.0 & $* *$ & 11 \\
& Residual & 20 & 0.9 & & & 16
\end{tabular}


Table S4. Results of the pairwise comparison testing for differences in the biochemical composition (in terms of protein. carbohydrate. lipid and phytopigment contents) between trawled and untrawled sites at each depth. $\mathrm{df}=$ degrees of freedom; MS = mean square; Pseudo-F = permutational F; significance of test obtained from Monte Carlo simulations: $*=p(\mathrm{MC})<0.05 ; * *=p(\mathrm{MC})<0.01 ; * * *=p(\mathrm{MC})<0.001$; n.s. $=$ not significant .

Pair-wise comparisons

Factors: Trawled vs.Untrawled

\begin{tabular}{llll} 
& Depth $(\mathrm{cm})$ & $\mathrm{t}$ & $p(\mathrm{MC})$ \\
\hline Biochemical composition & $0-1$ & 2.237 & n.s. \\
$1-3$ & 5.353 & $* *$ \\
$3-5$ & 2.456 & $*$ \\
$5-7$ & 2.565 & $*$ \\
$7-9$ & 2.875 & $*$
\end{tabular}

Table S5. Results of the PERMANOVA testing for differences in (a) relative contribution of phytopigments to biopolymeric C and (b) protein $\mathrm{C}$ turnover rates between examined factors and their interaction. The pairwise comparison testing for differences of each investigated variable between trawled and untrawled sites at each depth is also included. $\mathrm{df}=$ degrees of freedom; MS = mean square; Pseudo-F = permutational F; significance of test obtained from Monte Carlo simulations: $*=p(\mathrm{MC})<0.05 ; * *=p(\mathrm{MC})<0.01 ; * * *=p(\mathrm{MC})<0.001$;

10 n.s.= not significant.

(a)

Pair-wise comparisons

\begin{tabular}{|c|c|c|c|c|c|c|c|c|c|}
\hline & \multirow[b]{2}{*}{ Source } & \multirow[b]{2}{*}{ df } & \multirow[b]{2}{*}{ MS } & \multirow[b]{2}{*}{ Pseudo-F } & \multirow[b]{2}{*}{$p(\mathrm{MC})$} & \multirow[b]{2}{*}{$\begin{array}{c}\text { Explained } \\
\text { variance }(\%)\end{array}$} & \multicolumn{3}{|c|}{ Factors: Trawled vs.Untrawled } \\
\hline & & & & & & & Depth $(\mathrm{cm})$ & $\mathrm{t}$ & $p(\mathrm{MC})$ \\
\hline \multirow[t]{3}{*}{ Phytopigment/BPC (\%) } & Site & 1 & 3.6 & 59.1 & $* * *$ & 18 & $0-1$ & 1.438 & n.s. \\
\hline & Site x Depth & 4 & 0.1 & 1.3 & n.s. & 0 & $3-5$ & 6.698 & ** \\
\hline & Residual & 20 & 0.1 & & & 5 & $5-7$ & 4.143 & $*$ \\
\hline
\end{tabular}

(b)

Pair-wise comparisons

Factors: Trawled vs.Untrawled

\begin{tabular}{|c|c|c|c|c|c|c|c|c|c|}
\hline & Source & df & MS & Pseudo-F & $p(\mathrm{MC})$ & $\begin{array}{c}\text { Explained } \\
\text { variance }(\%)\end{array}$ & Depth $(\mathrm{cm})$ & $\mathrm{t}$ & $p(\mathrm{MC})$ \\
\hline \multirow[t]{4}{*}{ Turnover $\left(10^{-3} \mathrm{~d}^{-1}\right)$} & Site & 1 & 1.2 & 26 & $* * *$ & 5 & $0-1$ & 5.400 & $* * *$ \\
\hline & Depth & 4 & 5.5 & 120.4 & $* * *$ & 64 & $1-3$ & 5.452 & $* *$ \\
\hline & Site $x$ Depth & 4 & 1.2 & 26.4 & $* * *$ & 27 & $3-5$ & 4.242 & $*$ \\
\hline & Residual & 20 & 0 & & & 3 & $5-7$ & 1.234 & n.s. \\
\hline
\end{tabular}

